Vivre, penser, écrire en exil

\title{
Bouhout Abdelkrim, Essai sur la visibilité des migrants relégués
}

Brahim Labari

\section{(2) OpenEdition \\ 12 Journals}

Édition électronique

URL : https://journals.openedition.org/remi/8495

DOI : $10.4000 /$ remi.8495

ISSN : $1777-5418$

Éditeur

Université de Poitiers

\section{Édition imprimée}

Date de publication : 1 mars 2017

Pagination : 165-167

ISBN : 979-10-90426-30-6

ISSN : 0765-0752

\section{Référence électronique}

Brahim Labari, «Bouhout Abdelkrim, Essai sur la visibilité des migrants relégués », Revue européenne des migrations internationales [En ligne], vol. $33-n^{\circ} 1$ | 2017, mis en ligne le 01 mars 2017, consulté le 16 avril 2022. URL : http://journals.openedition.org/remi/8495; DOI : https://doi.org/10.4000/remi.8495 


\section{Notes de lecture}

\section{Bouhout, Abdelkrim}

Essai sur la visibilité des migrants relégués. Paris : L'Harmattan, 2015. - $216 \mathrm{p}$.

ISBN : 978-2-3430-6395-9

Dans un ouvrage de 216 pages, Abdelkrim Bouhout se penche sur la condition du migrant relégué dans le contexte migratoire belge. Cette figure de la migration n'a pas fait l'objet d'une abondante littérature. Pour la traiter, I'auteur déploie un regard anthropologique en se situant, par le truchement de quelques faits rapportés et analysés, près de l'expérience urbaine de ces migrants, et en mobilisant d'énormes références bibliographiques. L'imaginaire traverse de bout en bout le livre à telle enseigne qu'on le retrouve dans l'intitulé des trois chapitres qui scandent inégalement l'ouvrage.

Le premier chapitre, "L'imaginaire assiégé ou la pensée captive de l'humiliation ", tente de charger de sens le concept de relégation pour lui trouver une sorte d'extension empirique. Ce cours chapitre (pp. 13-41) peut être appréhendé comme une entrée dans le sujet qui manifestement interpelle l'auteur. Un sujet à la fois sous-traité dans la littérature "immigrée ", mais pénétré d'un sens commun têtu : les relégués sont représentés comme faisant partie des "classes populaires" dangereuses qui mettraient à rude épreuve le paradigme du vivre-ensemble. Au commencement de son propos, l'auteur donne ainsi le ton : "Au premier regard, le multiculturalisme bruxellois est décent. On mange bien à Bruxelles. Les mets et les gourmets se bousculent, le folklore domine. Quelle démocratie que celle des mœurs ! La vraie comme l'entendait Baudelaire. Toutefois, par-delà ce folklore d'idées grouillantes, le vivre-ensemble est loin d'être de tout repos » (p. 9). Plus précisément, la dimension empirique de l'exer- cice apparait clairement quand l'auteur a administré un nombre conséquent de questionnaires qu'il a analysés dans le but de mieux appréhender les représentations sociales autour du migrant relégué, c'està-dire déclassé en prenant appui sur le cas de l'entreprise (pp. 28-34). Les travaux de Pascal Moliner lui ont servi de guide et de modèle, qui argue que « le système central, structurant les cognitions relatives à l'objet est le fruit des déterminismes historiques, symboliques et sociaux auxquels est soumis le groupe social " (p. 28). Cette méthode use de trois questionnaires dont les deux derniers font fonction "d'indice ". En résumé, l'usage d'un " indice de réfutation " permet de divulguer scientifiquement le noyau sémantique et de divulguer le sens de la représentation de l'entreprise au-delà des postures, notamment celles convoquées par les classes moyennes dotées d'un capital culturel conséquent.

C'est en réaction à la vox populi que l'auteur commet son texte en reconvoquant sa propre expérience et en ayant fait le choix d'une appréhension anthropologique du phénomène de la relégation. II rappelle à juste titre qu'entre les deux modèles extrêmes, l'anglo-saxon prônant sans complexe une société multiculturelle et le jacobinisme français visant l'assimilation républicaine de ses migrants, il y a sans doute à réinventer une troisième voie, celle d'un migrant modulé sur la base d'une double expérience " anthropologique " : en tant qu'arraché à son terroir d'origine et comme propulsé dans la ville et subissant par voie de conséquence la politique de la gestion de la diversité. Cette posture de l'auteur rappelle celle de Robert E. Park décrivant la condition et l'expérience écologique de The marginal man à partir de la dimension subjective de l'expérience migratoire. 
Le deuxième chapitre " L'imaginaire errant ou l'identité contrapuntique ", appréhende le migrant relégué à la fois comme objet-réceptacle de tous les maux que les autres projettent sur lui : bâtard et inutile, voire une charge pour les travailleurs sociaux. Mais le migrant relégué résiste aussi aux tentatives de stigmatisations dont il s'érige en objet tout désigné pour gérer une indétermination identitaire des plus aiguës : " c'est par le bon aloi de son hybridité, que l'exil, cette expérience particulière de l'incomplétude se défait des essais, esquisses et œuvres littéraires enferrant le fait migratoire aux préjugés culturels et raciaux " (p. 45). Le corps est alors comme un atout à mettre en scène. Ce que l'auteur a pris dans la tradition de Chicago, c'est la notion " d'aire morale " (moral area). Effectivement, les manières de faire la ville font des manières d'être en ville et l'évolution migratoire dans des espaces relégués de la capitale produit un tempérament. Combiné à l'incomplétude identitaire héritée de l'expérience migratoire, ce tempérament débouche sur une vision du monde marquée par l'avanie. Dès lors, l'imaginaire assiégé (par l'avanie) et l'imaginaire contrapuntique (incomplétude identitaire) justifient une anthropologie culturelle de la relégation.

Le dernier chapitre " L'imaginaire oxymorique ou l'identité paradoxale ", plus long et consistant, fait un arrêt sur le concept de l'identité appréhendé dans une perspective holiste, inspirée par l'anthropologie culturelle. Ce chapitre est l'occasion pour l'auteur de déployer son regard sur l'une des questions les plus problématiques en sciences sociales, celle de l'identité et des stratégies pour la rendre visible. Comme dans pratiquement tout le texte, l'auteur nous entraîne dans des tours et détours de la conceptualisation par le bas en allant chercher des références dans des registres aussi éloignés que la littérature, la philosophie ou la psychanalyse. En deux paragraphes, l'auteur loge à la même enseigne Arendt, Camus, Carassus, König de l'École de Cologne, pour affirmer que la visibilité est le fondement ontologique des êtres humains, mais qui revêt dans le contexte " arabe " le simulacre du sujet " empharé » (p. 84). Que veut en définitive I'auteur, au-delà de son vœu méthodologique, celui de tailler une assise anthropologique au migrant relégué ?

Au bout d'une lecture hardie, l'ouvrage peut être rattaché à l'anthropologie empathique entendue comme un processus de connaissance réflexif, autrement dit résultant d'un rapport filial assemblant l'auteur et son objet d'étude pour ne pas dire son sujet. Qui est l'auteur et pourquoi $s^{\prime}$ est-il saisi de cet objet à la fois méconnu et prégnant dans les cultures urbaines bruxelloises ? De quelle légitimité dispose-t-il pour mener un tel travail ? L'anthropologie est la science de l'Homme, dit-on, science de l'Homme dans sa totalité et son unité, mais également dans la diversité des cultures qui caractérise la vie humaine. L'auteur, comme le dit la quatrième de couverture, est enfant d'immigré marocain et citoyen belge. Cette bi-appartenance lui confère à l'évidence une posture privilégiée pour étudier ces migrants-relégués à la manière de Richard Hoggart dans sa " culture du pauvre ".

L'ouvrage est donc bâti sur un objet : le migrant relégué qui a donc toutes les allures d'un marginal aux multiples appartenances, vivant dans le provisoire et l'imprévisibilité, accablé par sa condition d'ancien colonisé, en l'occurrence ici le Maghrébin, ciblé par des représentations stigmatisantes en contexte belge. L'auteur fait sienne la posture anthropologique s'attachant à comprendre le migrant qui, arraché à sa terre natale et de prime socialisation, est jeté dans l'univers urbain auquel il est astreint de s'acculturer. Faute de quoi, le déclassement serait sa destinée.

Manifestement à l'aise dans le frottement au sens commun dans la perspective de l'ethnométhodologie, l'auteur expose narrativement ses expériences de terrain, toutes choses vues, vécues dans 
des configurations d'en bas et les remonte au " global ", au macro - " culture arabe ", " identité islamique ", " expérience totalitaire " - prenant appui sur des travaux sociologiques ou historiques (Hannah Arendt, Erving Goffman, Ibn Khaldoun, Daniel Rivet, etc.).

L'inflation des références, la pléthore des concepts et finalement cet éclectisme " paradigmatique " rendent la lecture de cet ouvrage assez déroutante. L'auteur, lui-même ouvert sur les deux cultures du nord et du sud de la Méditerranée, prendrait " son miel là où il le trouve ". La réflexivité est en effet une grande chose, on ne choisit pas spontanément les objets dont on se saisit !

En résumé, le livre comporte une thèse qui peut être résumée ainsi : la relégation urbaine produit des comportements qui sont autant de fruits du déclassement urbain et de l'expérience migratoire (migrant-relégué) homologuant le concept de disruption sociale. Cette disruption qualifie les conduites du migrant-relégué à trois niveaux. Elle use de la visibilité et des masques sociaux (d'où la pertinence de Erving Goffman et de l'École de Cologne). Sur le plan de l'identité individuelle, la visibilité ostentatoire réhabilite le sentiment de dignité. Sur le plan de l'identité sociale, la disruption produit des " narrations " qui restaurent l'identité groupale. Enfin sur le plan symbolique, la disruption sociale est une thermodynamique qui régule l'économie affective : reconfiguration de I'ancien (mœurs paysannes) au contact du nouveau (société de consommation). Là réside essentiellement les conduites oxymoresques, sorte d'alliance truculente du nouveau et de l'ancien chez le migrantrelégué.

Nous devrions savoir gré à Abdelkrim Bouhout d'avoir enrichi la littérature sur les migrations d'un pavé de bonne facture où se mêlent un exercice de style, une sorte d'anthropologue comme écrivain à la Geertz, et la vivacité d'un dispositif argumentaire assez huilé.

Brahim Labari

Professeur de sociologie

Sociologie des Organisations et Mutations Socio-économiques/Université d'Agadir

\section{Longo, Teresa Mariano \\ Roche, Thierry \\ L'enfance à l'école des autres. Un regard. - Paris : Téraèdre, 2015. - $198 \mathrm{p}$. \\ ISBN : 978-2-3608-5065-5}

Comment des enfants d'origines diverses vivent-ils ensemble au sein d'une classe? C'est à cette question que l'ouvrage tente de répondre au moyen de deux enquêtes ethnographiques réalisées par un anthropologue spécialisé en cinéma et une chercheure en éducation comparée, qui ont travaillé pendant trois ans dans deux classes d'école primaire à Cirta en Roumanie et à Ferrare en Italie. Plus largement, ce travail s'appuie sur un projet porté par l'équipe pluridisciplinaire Habiter le monde, de I'Université de Picardie Jules Verne, qui vise à comprendre comment les enfants d'origines culturelles diverses vivent ensemble dans l'école.

L'intérêt de ce livre est pluriel. Tout d'abord, l'étude de deux contextes sociohistoriques différents permet de décentrer le regard pour chercher une réponse à des questions communes sur l'école et l'enfance. Les deux chercheurs partagent une démarche résolument inductive, compréhensive et qualitative qui permet à la problématique de se préciser au cours de la recherche. Cette approche comporte l'avantage de pouvoir s'adapter aux aléas du terrain et de conserver un esprit assez ouvert pour ne négliger aucune explication ou direction, et ainsi de rendre compte des expériences vécues dans leur complexité. Elle favorise également la comparaison de situations très différentes pour en tirer des conclusions plus générales. Ainsi, le rapport à l'altérité diffère fortement entre 
la Roumanie et I'Italie, ce qui n'empêche pas que les deux terrains se nourrissent réciproquement au cours de l'enquête. Les rapports majoritaire/minoritaire connaissent de vives tensions en Roumanie qui se traduisent par de fortes compétitions entre groupes ethniques, dans lesquelles les populations rroms et tsiganes sont désavantagées. Le rapport aux migrants est également difficile dans ce pays qui découvre la question migratoire dans les années 1990, après la chute du régime de Ceaucescu. Tout en étant un pays de forte émigration vers l'Ouest, la Roumanie est devenue en l'espace de deux décennies un espace de transit et d'arrivée pour des migrants aux profils variés ${ }^{1}$. Les tensions qui s'expriment dans la société roumaine, eu égard aux populations minoritaires et migrantes, se donnent à voir dans la classe enquêtée qui fonctionne comme un microcosme social dans lequel les Rroms sont fortement stigmatisés. Les sensibilités sont vives entre les Roumains, les Saxons, les Magyars et les Rroms et les auteurs font le constat d'une " école qui continue de célébrer les distinctions et les séparations sociales " en institutionnalisant la ségrégation entre les élèves. D'ailleurs, les difficultés à enquêter décrites dans la première partie de l'ouvrage révèlent bien les difficultés du groupe à faire classe, à " habiter le lieu ensemble ". Plus encore, le recours systématique de l'école à la police, présente lors des récréations et à la sortie des classes, est particulièrement révélateur des tensions ethniques. La forte hiérarchisation de la société roumaine se traduit par un modèle de différenciation sociale dont les auteurs montrent bien qu'il est reproduit à l'école.

Le contexte migratoire de l'Italie connaît quelques similitudes contextuelles, dans la mesure où les migrations internes se sont longtemps traduites par le départ des

1 Michalon Bénédicte et Nedelcu Mihaela (2010) La Roumanie et ses migrations, vingt ans après la chute du communisme, Revue d'Etudes Comparatives Est-Ouest, 41 (4), pp. 5-27. populations du sud vers le nord, ce qui n'a jamais été sans heurts comme en atteste notamment la montée en puissance de la Ligue du Nord. De même, I'Italie a connu un bouleversement de la balance migratoire avec des flux d'immigration de plus en plus importants, légaux comme illégaux, à partir des années 1980-1990. Toutefois, les options politiques retenues ont été plus favorables qu'en Roumanie, avec plusieurs vagues de régularisation et, à l'école, une volonté politique et pédagogique inclusive des populations migrantes. Particulièrement révélatrice des tensions sociales que connaissent les deux pays, la comparaison entre ces deux terrains permet d'éclairer la question du vivre ensemble sous l'angle du rapport à l'altérité dans le cadre scolaire et au sein des relations enfantines.

L'autre intérêt majeur de cette comparaison réside dans le rapport à l'enfance des sociétés roumaine et italienne. Pour les appréhender, les deux auteurs font le pari d'une démarche ethnographique qui, outre l'observation du rapport à l'altérité, permet de s'immiscer dans l'entre-soi de la classe afin d'observer les relations entre pairs ainsi que le rapport enfants/adultes et maître/élève. Surtout, l'originalité de la démarche est d'associer l'anthropologie visuelle aux méthodes d'observation en classe, en s'appuyant sur les écueils d'une recherche précédente menée dans des classes à Beauvais et à Naples où l'image n'était finalement assujettie qu'à sa seule fonction émotionnelle. Cette fois, il s'agit pour les deux chercheurs d'accompagner l'image par du texte qui restitue l'expérience migratoire, ce qui constitue l'objet même de l'ouvrage. L'anthropologie visuelle est utilisée pour " filmer l'école comme un théâtre et la classe comme une scène " pour saisir les interactions entre l'enseignante et les enfants et répondre à quelques questions parmi lesquelles "Quand et comment les enfants font-ils groupe ? ", "Quand l'école devient-elle un lieu de l'enfant et de l'enfance où des enfants nouvellement arrivés et d'origines 
diverses peuvent se retrouver avec des pairs ? ", "Comment l'école, les adultes, les savoirs influencent les manières d'être du groupe d'enfants et vice-versa? "..

Les images de l'enfance divergent là aussi selon les deux pays. Le visionnage des films tournés dans les deux classes permet de mieux appréhender ces différences en revenant sur des situations de classe révélatrices de rapports de domination construits différemment dans les deux pays. Le contenu des cours, la méthode utilisée et la culture scolaire que les enseignants transmettent projettent les enfants vers deux modèles de société distincts selon les auteurs. Dans la classe de Cirta en Roumanie, l'autorité est confondue avec la force et les problèmes sociaux sont rapportés à des problèmes moraux sur le mode " il y a des gens qui se comportent mal, il faut les expulser ou les interner ". Dans la classe de Ferrare en Italie, l'image révèle que l'espace appartient à tous, les élèves disposant à leur guise du bureau de l'enseignante, de l'armoire, du tableau, du matériel. Au risque parfois d'opposer deux visions diamétralement opposées, l'une obscure et l'autre naïvement idyllique, les deux auteurs révèlent néanmoins de façon convaincante les écueils et les avancées de deux sociétés face à leurs enfants et à leurs minorités/migrants.

Au final, cet ouvrage apporte un éclairage important sur le rapport à l'altérité tout autant que sur le rapport à l'enfance de deux sociétés européennes occupant des positions spécifiques sur la scène migratoire européenne. L'approche inductive, la démarche participative et I'utilisation de I'anthropologie visuelle fonctionnent comme des valeurs ajoutées à l'enquête ici restituée, afin de mieux comprendre les rapports inter-ethniques et intergénérationnels. Par ailleurs, les longues descriptions de lieux qui témoignent du fort intérêt de Thierry Roche pour le paysage et l'urbanisme apportent un éclairage utile concernant leur impact sur les relations sociales. On regrette toutefois le peu de place accordée au contexte socio-politique des deux pays dans lesquels les enquêtes de terrain ont été menées, hormis quelques pages d'annexes. De même, le choix de placer en fin d'ouvrage une partie sur le traitement de l'enfance dans les sciences sociales, bien que bien documentée, contribue à un effet de juxtaposition qui dessert quelque peu la cohérence d'ensemble.

\section{Isabelle Rigoni \\ Chercheure, Centre Émile Durkheim (UMR-CNRS, Bordeaux) \\ Maître de conférences en sociologie, INS HEA Suresnes}

\section{Khaknégar, Nahâl \\ L'exil comme épreuve littéraire. L'écrivain iranien face à ses homologues. - Paris : \\ L'Harmattan, 2015. - $246 \mathrm{p}$. \\ ISBN : 978-2-3430-5571-8}

Nahâl Khaknégar appuie son étude sur deux romans récents qui ont remporté un grand succès international, Le Voyage des bouteilles vides (2001 en traduction française) écrit en néerlandais par l'Iranien Kader Abdolah et Les Belles choses que porte le ciel (2007) publié en anglais par I'Ethiopien Dinaw Mengestu. Les deux romanciers choisis sont des émigrés qui ont emprunté le chemin de l'intégration et rédigé dans une langue différente de leur idiome maternel.

L'ouvrage s'ouvre par des généralités portant sur l'histoire, la sociologie et la psychologie de la migration ainsi que sur la littérature inspirée par ce phénomène, particulièrement en France. Les pages consacrées à ces thèmes sont les moins convaincantes du livre, car il se révèle impossible de traiter de manière nuancée et exhaustive des sujets aussi riches et complexes. Aussi les lacunes, les raccourcis, voire les banalités, caractérisent-ils cette partie. Dans le cas de la France, I'auteur cite essentiellement des ouvrages dus aux réfugiés allemands de 
l'entre-deux-guerres et aux Maghrébins de la période suivante, mais les Russes blancs et les Arméniens qui ont beaucoup écrit ne sont pas cités, ni, dans le cas des exilés volontaires, les Américains de la "Génération perdue ". En outre, cette partie, construite sur des énumérations et des micro-paragraphes semblant traduire les difficultés éprouvées par l'auteur pour rendre compte d'une matière trop ample, souffre d'une construction maladroite.

Le livre se développe sur un mode plus positif quand il aborde le statut de l'écrivain iranien en exil, généralement ancien acteur de la Révolution dans son pays, déçu par l'effondrement de ses espoirs progressistes et l'avènement d'un régime islamiste, marginalisé loin de ses racines, éprouvant souvent un obscur sentiment de culpabilité, essayant de se libérer du carcan des traditions anciennes pour aller à la rencontre d'une autre culture ou même de l'essence de l'humanité. L'ouvrage se poursuit par deux monographies analysant chacun des romans autofictionnels constituant le corpus choisi. L'espace occupé par les personnages, leur rapport au temps, leurs rencontres, surtout avec des marginaux comme eux, leurs souvenirs et leurs espoirs, leur volonté plus ou moins forte d'intégration sont passés en revue. L'ouvrage s'achève par une comparaison entre les deux romans dont les thématiques apparaissent proches : espoir du retour, problèmes de langue, recherche d'une utopie pouvant remplacer la terre natale, obsession de l'intégration, omniprésence du mouvement comme métaphore de l'exil. La conclusion contredit un peu le sous-titre du livre qui met en valeur l'écrivain iranien ; ici, l'écrivain éthiopien apporte autant au bilan final. Et celui-ci se révèle intéressant : le roman d'immigration contemporain qui synthétise divers imaginaires dépasse le traditionnel questionnement porté sur le monde et les rapports noués avec l'Autre. L'écrivain cherche à savoir qui il est, quelle identité s'est formée en lui au fil des épreuves. Nahâl Kahknégar observe : "Pour l'immigré d'aujourd'hui, la question n'est plus "Où est ma patrie ?", mais "Qui suis-je ?" ". Les romans ne présentent guère de héros, mais des personnages dont l'individualité se trouve valorisée au détriment de l'appartenance collective.

Ralph Schor

Professeur émérite d'histoire Université de Nice-Sophia-Antipolis

\section{Lacroix, Thomas}

Hometown Transnationalism, Long Distance Villageness among Indian Punjabis and North African Berbers. - Basingstoke : Palgrave Macmillan, 2016. - 217 p. ISBN : 978-1-1375-6720-8

Le livre de Thomas Lacroix est appelé à devenir un ouvrage de référence sur le transnationalisme villageois. À mi-chemin entre l'étude empirique et la réflexion épistémologique, il démontre que l'engagement transnational est une réponse collective faite par des migrants à la question " qu'est-ce qu'être un villageois ? " (p. 178). L'ouvrage tente de saisir le point de jonction entre les processus de développement et les associations villageoises transnationales en trois temps. Tout d'abord, l'auteur présente le cadre méthodologique et théorique de sa réflexion, pour, ensuite, décrire et conceptualiser le processus de construction des organisations transnationales villageoises. Enfin, la troisième partie examine le rôle joué par les contextes politiques des pays d'installation (Royaume-Uni et France) d'une part et des pays de départ (Maroc, Algérie, Inde), d'autre part, sur ces dynamiques transnationales. Cet ouvrage est original et pertinent, car il est bâti sur un travail comparatif rigoureux et inédit, réalisé sur plus d'une décennie, entre trois groupes de populations immigrées en Europe occidentale : les Punjabis (Indiens), les Chleuhs (Marocains) et les Kabyles (Algériens). II

2 Les traductions sont faites par l'auteur de la recension. 
allie enquêtes de terrain et travail quantitatif et adopte une démarche épistémologique hybride, entre géographie, sociologie et sciences politiques. Ensuite, il mobilise une littérature qui fait la part belle aux études transnationales nord-américaines littérature que l'auteur a particulièrement contribué à diffuser dans l'espace académique français.

Nous retenons plusieurs apports significatifs en matière de conceptualisation des initiatives transnationales de développement portées par des migrants. En premier lieu, Thomas Lacroix propose une définition des organisations villageoises de développement (ou HTOs, Hometown Organisations) précise qui échappe aux catégories de la pratique. II poursuit ce que ses travaux antérieurs avaient déjà affirmé : les organisations transnationales villageoises constituent des institutions sociales migrantes qui participent à la résolution des conséquences de multipolarisation de l'identité de leurs membres. Plusieurs aspects de cette définition méritent d'être soulignés. D'abord, I'auteur affirme que les HTOs ne sont pas des structures figées, mais plutôt des organisations douées d'une grande capacité à incorporer le changement social. Ce faisant, elles participent à l'élaboration d'une « villageoisité "(villageness), qui serait le produit de l'articulation entre appartenance villageoise, appartenance globale et processus de construction identitaire (p. 63). Celle-ci permet, pour chacun des membres des HTOs, d'opérer un point de connexion entre un "nous " et un " eux " (p. 88). Troisièmement, cette définition s'appuie sur l'idée de "spatialité morale ", selon laquelle il existerait un espace moral de la migration (avec son ensemble de devoirs associés), dans une relation étroite entre identité villageoise et spatialité. Ainsi, les HTOs mettent en place une " gouvernementalité longue-distance ", en transposant un cadre normatif qui donne un sens à la migration, mais permet également à la communauté villageoise de sécuriser le maintien des relations longue-distance et de réguler les flux (pp. 74-75). Ces éléments permettent à Thomas Lacroix d'affirmer que les organisations villageoises transnationales participent à la production d'une normativité morale néo-traditionnelle. Ce travail fin autour de l'économie morale de la "villageoisité " transnationale nous paraît être l'un des apports principaux de I'approche des HTOs proposée par l'auteur, dans une perspective qui tente d'éviter toute forme d'essentialisation des communautés transnationales.

Ce faisant, Thomas Lacroix prend position dans le champ des études transnationales au sein duquel il s'inscrit. II regrette son évolution contemporaine qu'il juge " préoccupante, par rapport à ses prémisses initiales " (p. 182), constate ses difficultés à prendre en compte l'imbrication des échelles et des facteurs matériels et immatériels, et déplore le fait que la dimension spatiale des processus transnationaux a concentré l'attention des chercheurs au détriment de la dimension temporelle. Ceci le conduit à proposer une nouvelle approche épistémologique combinant étude des structures sociales et de l'agentivité. Selon lui, les pratiques transnationales des migrants doivent être comprises comme une forme d'agentivité insérée simultanément dans plusieurs espaces sociaux et dans plusieurs univers structurels (p. 57). II est nécessaire de les étudier sur le temps long, en observant le processus d'adaptation des organisations associatives aux nouvelles caractéristiques des populations immigrées. Au-delà, I'auteur propose une lecture habermassienne d'objets centraux de l'étude des migrations et post-migrations, comme les transferts ou l'engagement en faveur du développement, et envisage toute migration comme un acte politique (pp. 71-72) déployé par un groupe social pour résister ou s'adapter à l'entropie (p. 82).

Cette démarche épistémologique soulève plusieurs interrogations et ouvre des pistes de recherche intéressantes. 
Par exemple, Thomas Lacroix rejette le " construit académique et politique rassurant " (p. 146) selon lequel l'incorporation des immigrés dépendrait exclusivement de l'agentivité étatique - tout en reconnaissant que les discours et l'action étatiques jouent un rôle dans la formation des comportements transnationaux (pp. 183-185). L'engagement transnational dans une HTO dépendrait davantage des conditions qui ont façonné la migration (p. 61) et de processus liés aux groupes migrants, comme la formation des familles dans les pays d'installation ou la diversification générale des statuts socio-professionnels (p. 95). Pourtant, si la structure de ces populations immigrées en France ou en Royaume-Uni a changé, c'est bien en partie sous l'effet des politiques nationales (migratoires, sécuritaires, d'intégration ou de développement) dont on aurait, d'ailleurs, aimé mieux comprendre comment l'auteur envisageait l'articulation et les effets respectifs et cumulés. Peut-on, dès lors, les mettre à distance dans l'analyse? Le travail deThomas Lacroix suscite l'envie de poursuivre la recherche dans deux directions : d'une part, l'étude des rapports de pouvoir entre les différents groupes d'acteurs qui participent à la construction des pratiques et des politiques transnationales ; d'autre part, l'étude des reconfigurations de la catégorie analytique des HTOs. En effet, les changements structurels de la composition de la population migrante, soulignés par l'auteur, ont nécessairement des conséquences en matière de déplacement de frontières entre la catégorie des HTOs et d'autres catégories proches que l'auteur mobilise (comme celle des ONG de migrants), ainsi qu'au sein de la catégorie HTOs elle-même. Plus précisément, on aimerait mieux comprendre le paradoxe apparent entre l'accroissement de la part des migrants urbains et le maintien de structures centrées sur la construction d'une " villageoisité " transnationale. Le lecteur s'interroge : au-delà de leur marginalité quantitative (soulignée par l'auteur), les HTOs seraient-elles une pratique déclinante?

Ainsi, cet ouvrage contribue de manière déterminante à I'amélioration de la connaissance scientifique des dynamiques associatives transnationales migrantes, en proposant une démarche épistémologique nouvelle pour les analyser et de nouveaux questionnements passionnants. Nous nous permettrons, en guise de conclusion, de plaider pour sa publication rapide en français, afin qu'il puisse contribuer au renouvellement des travaux sur les dynamiques transnationales migrantes.

Claire Vincent-Mory IEDES/Université Paris 1 Panthéon Sorbonne 